\title{
Honey Bee Venom Re-Challenge During Specific Immunotherapy: Prolonged Cardio-Pulmonary Resuscitation Allowed Survival in a Case of Near Fatal Anaphylaxis
}

Sara Micaletto ( $\sim$ sara_micaletto@hotmail.com )

University Hospital Zurich: UniversitatsSpital Zurich https://orcid.org/0000-0002-2187-8221

Kurt Ruetzler

University Hospital Zurich: UniversitatsSpital Zurich

Martin Bruesch

University Hospital Zurich: UniversitatsSpital Zurich

Peter Schmid-Grendelmeier

University Hospital Zurich: UniversitatsSpital Zurich

\section{Case report}

Keywords: specific immunotherapy, honey bee, re-challenge, anaphylaxis, cardio-pulmonal resuscitation

Posted Date: December 29th, 2021

DOI: https://doi.org/10.21203/rs.3.rs-1156492/v1

License: (c) (1) This work is licensed under a Creative Commons Attribution 4.0 International License.

Read Full License 


\section{Abstract}

BACKGROUND Specific immunotherapy for patients with honey bee hypersensitivity is commonly applied. Re-challenge with venom is performed to prove protection.

CASE PRESENATION We report a case of near fatal anaphylaxis with asystolia for 24 minutes in a 35years-old patient with mastocytosis after honey bee sting challenge despite 5-years of specific immunotherapy. Successful cardio-pulmonary resuscitation (CPR) was applied for 32 minutes.

CONCLUSION This intervention demonstrates, that in anaphylaxis with cardio-vascular arrest prolonged CPR for up to 40 minutes might be appropriate to overcome half time span of massively released histamine. Failure of specific immunotherapy was possibly due to sensitization to the allergen Api m10, probably underrepresented in commercial honey bee venom extracts. Also, molecular analyses might alert to potential unsuccessful outcome of venom specific immunotherapy especially in high-risk patients such as mastocytosis.

\section{Background}

Hypersensitivity to insect venom is a quite common cause for severe partly even life-threatening anaphylaxis and affects up to $8.9 \%$ of the population. 1 Honey bees (Apis mellifera) or yellow jackets (Vespula germanica and Vespula vulgaris) lead to systemic anaphylactic sting reactions in the majority of cases. ${ }^{2}$ Mastocytosis is a risk factor for very severe anaphylactic reactions in patients allergic to hymenoptera. ${ }^{3}$ Venom immunotherapy (VIT) is highly effective in preventing further systemic anaphylactic sting reactions, however, $2.4-20.4 \%$ of those allergic to bee venom or yellow jacket venom were not protected by $\mathrm{VIT}^{4}{ }^{4}$ Therefore, re-challenge is of importance to prove protection. ${ }^{5}$

\section{Case Presentation}

We report on a 35 year old patient, who experienced a severe anaphylactic reaction after a honey bee sting in 2007 with hypotension and loss of consciousness (Grad IV reaction). Sensitization on bee venom (BV) was confirmed with specific IgE in serum (i1, $24 \mathrm{kU} / \mathrm{I}$ ) and intradermal test (positive prick test at 0.01 $\mathrm{ug} / \mathrm{I}$ ), while no specific IgE against wasp (i3) were detectable. Thus, venom specific immunotherapy (VIT) with bee venom extract was initiated in June 2008 and performed according to international guidelines with 100 '000 SQE every 4 weeks and was well tolerated by the patient.4,5

In January 2013 a follow-up control after almost 5 years of immunotherapy was performed. Specific IgE antibodies for bee venom (i1) decreased from 24 to $6 \mathrm{kU} / \mathrm{l}$, while specific IgG were raised as expected after 5 years of VIT (see table 1). Unexpectedly serum tryptase level, in normal range in 2008 (3.3 ug/l) was now elevated to $21 \mathrm{ug} / \mathrm{l} .6,7$ Thus further investigations revealed some teleangiectesia on the tronc and swelling on mechanical pressure (positive Darier sign), a skin biopsy showed increased numbers of mast cells compatible with cutaneous mastocytosis.7 Except of sporadic dizziness no symptoms compatible with systemic mastocytosis were reported by the patient; c-Kit mutation was not detectable. 
However, a bone marrow aspirate showed two minor criteria of systemic mastocytosis (increased number of abnormal mast cells $>25 \%$, expression of aberrant CD 25 cells). 8

Table 1: Patient serum analysis on molecular sensitization patterns

Patient serum analysis (time of sting provocation)

\begin{tabular}{|llllll|}
\hline & rApi $\mathbf{m} 1$ & rApi m2 & rApi m3 & rApi m4 & rApi m10 \\
\hline $\mathbf{l g E ~} \mathrm{kU} / \mathbf{I}$ & 89.2 & $>100$ & 1.85 & 9.94 & 10.5 \\
\hline $\mathbf{l g}$ 4 $\mathbf{u g} / \mathbf{l}$ & $>50000$ & 7285 & 272 & $>50000$ & 241 \\
\hline
\end{tabular}

Comparison data: serum of a protected patient

\begin{tabular}{|c|c|c|c|c|c|}
\hline & rApi m1 & rApi m2 & rApi m3 & rApi m4 & rApi m10 \\
\hline lgE kU/l & 1.6 & 15.9 & 0.22 & 2.3 & 2.42 \\
\hline lgG4 ug/l & $>50000$ & 15190 & 19320 & $>50000$ & 1800 \\
\hline
\end{tabular}

Patient serum analysis (time of sting provocation; 1 month later; after 6 months of VIT)

\begin{tabular}{|c|c|c|c|c|c|c|c|}
\hline & rApi m1 & rApi m2 & rApi m3 & rApi m4 & rApi m5 & rApi m10 & Date** \\
\hline lgE kU/l & 89.2 & $>100$ & 1.85 & 9.94 & 9.9 & 10.5 & $T+3$ days \\
\hline lgG4 ug/l & $>50000$ & 7285 & 272 & $>50000$ & 1890 & 241 & $T+3$ days \\
\hline lgG4 ug/l & $>50000$ & 7692 & 339 & $>50000$ & 2470 & 533 & $T+28$ days \\
\hline lgG4 ug/l & $>50000$ & 10640 & 1716 & $>50000$ & 3834 & $<200$ & $\mathrm{~T}+7$ months * \\
\hline lgG4 ug/l & & & & & & $<200$ & $\mathrm{~T}+4$ years $^{\circ}$ \\
\hline
\end{tabular}

$\left.{ }^{*}\right) 6$ months after reaching triple VIP maintenance dose (100'000 SQE plus 300 mg HBV concomitant with Omalizumab)

(**) T stands for day of sting challenge

${ }^{\circ}$ in between well tolerated 3years after sting challenge, under VIT mot Omalizumab and 200ug HBV

The patient was working as a gardener and therefore had a need to know his level of protection due to a high risk of re-stings - also knowing that patients with a tolerated sting challenge have a better quality of 
life.9 It is known that patients with mastocytosis are at higher risk for severe or even fatal anaphylaxis, after stopping or even during venom specific immunotherapy (VIT).10

Regarding the promising levels of decreased specific IgE and increased IgG4 against BV, we agreed finally on the patient will on a sting challenge with a honey bee, performed according to international guidelines for safety measurements to check whether protection by VIT was achieved and under ongoing VIT. Sting challenge was performed 10 days after last injection of maintenance dose. The patient was monitored with ECG, non-invasive blood pressure and pulse oximetry and intravenous access was established. The sting was performed on the patient's forearm; the stinger was left for $1 \mathrm{~min}$ in situ before being removed.

Four minutes later the patient reacted with a generalized flush and nausea, followed by cramps and vomiting. Despite immediately administered of two doses of $0.3 \mathrm{mg}$ of adrenaline given intramuscularly and $250 \mathrm{mg}$ of methylprednisolone as well as $2 \mathrm{mg}$ of clemastine intravenously, the condition of the patient worsened. He developed within minutes severe emesis and dyspnea, followed by tachycardia and hypotension. Chest compressions were immediately started, and the CPR (cardio-pulmonary resuscitation) in-house alarm was triggered. Two minutes later the in-house CPR team arrived initiated advanced CRP treatment. At this moment the SpO2 level was above $60 \%$, the patient showed signs of central hypoxia, non-invasive blood pressure could not be measured and central carotid pulse was extremely weak. Assisted ventilation was started. Simultaneously the ECG showed 2 nd degree AV block for about 30 seconds, followed by ventricular fibrillation. Defibrillator was attached and patient was endotracheal intubated. CPR was performed, strictly following international CPR guidelines.11,12 The patient had to be external defibrillated (biphasic with $200 \mathrm{~J}$ ) twice, followed by pulseless electric activity (PEA) for further 4 minutes. PEA limited and led to asystolia for a cumulative 24 minutes. Thereafter, ventricular fibrillation recurred, and patient was external defibrillated twice again. After a total of 32 minutes of CPR, intravenous administration of $10 \mathrm{mg}$ adrenaline and $2000 \mathrm{ml}$ isotonic fluid return of spontaneous circulation (ROSC) occurred. Amiodarone, fentanyl, rocuronium, propofol and midazolame was intravenously administrated and the patient was hemodynamically stabilized. The patient was transferred to the emergency department, where a central venous and an arterial catheter were inserted and a cumulative $1500 \mathrm{ml}$ isotonic fluid administrated (results of initial and before discharge arterial analyzes are summarized in Table 2.). The patient was finally transferred to intensive care unit, where therapeutic hypothermia for 24 hours was initiated. 
Table 2

Pathophysiologic changes during and after CPR

\begin{tabular}{|c|c|c|}
\hline & Initial analyze (time: 12:26) & Admission to ICU (time: 13:13) \\
\hline $\mathrm{pH}$ & 7.058 & 7.197 \\
\hline $\mathrm{pCO}_{2}(\mathrm{kPa})$ & 5.93 & 5.51 \\
\hline $\mathrm{pO}_{2}(\mathrm{kPa})$ & 26.1 & 28.4 \\
\hline Hkt (\%) & $0.543(*)$ & $0.548\left(^{\star}\right)$ \\
\hline $\mathrm{Hb}(\mathrm{mmol} / \mathrm{l})$ & 17.7 & 17.9 \\
\hline Lactat(mg/dl) & 11.2 & 6.2 \\
\hline Glucose (mmol/l) & 18 & 15.1 \\
\hline $\mathrm{HCO}_{3}(\mathrm{mmol} / \mathrm{l})$ & 11.9 & 15.5 \\
\hline Base excess & -19.5 & -12.4 \\
\hline Total fluid administration & $2000 \mathrm{ml}$ & $3500 \mathrm{ml}$ \\
\hline
\end{tabular}

The patient recovered slowly, initially showing transitory signs of "posttraumatic" such as reduced fine motor skills and concentration. However, during a several months rehabilitation program these steadily improved so that finally no permanent sequalae remained and the patient has returned to his former work.

The treatment of the patient was continued with Omalizumab and 300 ug honey venom extract every 4 weeks. With this treatment he seems to be protected as 3 years after the reported anaphylactic event the patient got stung again several times. Once he was stung simultaneously by two honey bees during his work; all stings were well tolerated. Interestingly, levels of IgG4 against Api m10 remained low.

\section{Discussion And Conclusion}

Duration of CPR theoretically partly corresponds to half-time of histamine to overcome circulation relevant effects.13,14 Maximal amounts of histamine found 20 to 30 minutes after challenge and were shown to correlate very well with the severity of anaphylaxis.15,16 Tryptase level two hours after sting challenge was measured and was excessively increased to around $1300 \mu \mathrm{g} / \mathrm{l}$, confirming an extreme degranulation of mast cells. 3 This fulfills all criteria of mast cell activation (MCA) requiring a $20 \%$ increase from physiological baseline tryptase levels.3 Four days after, tryptase level was fallen down to $10.4 \mathrm{ug} / \mathrm{l}$, then increased again to $16 \mathrm{ug} / \mathrm{l}$. 
Hymenoptera venoms typically contain a mixture of 3 to 4 major proteins as well as pharmacologically active peptides and other small molecules. There are common proteins but also significant differences amongst the various Hymenoptera species. 17 The possibility to measure specific IgE also on a molecular pattern has substantially contributed for a better understanding of allergologic mechanisms in recent years.18 A detailed analysis of molecular sensitization patterns was performed and showed a relevant sensitization not only to major honey bee allergens Api $\mathrm{m} 1$ and Api $\mathrm{m} 2$, but among other proteins also to Api m3 and an even higher level to Api m10 (see table 1). Earlier studies demonstrated that commercial extracts seem to contain only very limited amounts of Api $\mathrm{m} 10$, in contrary to natural honey bee venom. 19 In the present case we retrospectively found that after the 5 years course of VIT the patient did show increased IgG to Api $\mathrm{m} 1$ and Api m2, but not to Api $\mathrm{m} 10$ reflecting possible insufficient immune response to Api m10. Thus, after near fatal sting challenge VIT was up-dosed to finally 300 ug BV per session, using a part of $200 \mathrm{ug}$ in aquous solution of honey bee venom, which may contain higher amount of Api m10. In addition, the patient was put under preventive treatment with $\mathrm{H} 1$ - and $\mathrm{H} 2$-blockers, ketotifen and omalizumab. After 6 months of VIT with a total dose of 300 ug honey bee venom every 4 weeks (always with Omalizumab) the patient showed an increase of IgG4 also to Api m3 and Api m5 but still almost no increase to Api m10 - while in comparison a sting-challenged and protected mastocytosis patient showed relevant titers of Api m10 specific lgG4 (see Table 2). Thus, mismatch between allergens such as Api m10 might explain severe anaphylaxis occurring after a bee sting in mastocytosis patients despite performed VIT. As recently suggested for food allergens 20,21 , and seen in this case, lack of allergens might only be partly compensated by dose escalation if, extracts are not truly "spiked" with the relevant lacking allergens. In the obvious absence of protective IgG 4 antibodies against Api $\mathrm{m} 10$ the question is whether Omalizumab is protecting the patient by modulating the IgE-depended immune response or whether Api $\mathrm{m} 10$ was not playing an important role in the near fatal event after the sting challenge - as seen in other studies where patients with exclusively no IgG4 response to Api m10 also tolerated re-stings. Data from the use of Omalizumab as premedication for honey bee VIT with severe side effects underscore its potential therapeutic effect.22 Additionally, Omalizumab is reported to be protective in patients with mastocytosis and anaphylaxis and bee keepers not protected by conventional VIT with HB.23,24

We conclude the following for the clinical application of VIT: re-challenges to hymenoptera venom should only be performed by a trained stuff with all measures in place to start immediate CPR in case of severe anaphylaxis and all the resources to quickly escalate treatment to advanced CRP and ICU transfer; in case of anaphylaxis with cardio-vascular arrest prolonged CPR for up to 40 minutes might be appropriate to overcome half time span of released histamine and other cardio-vascular active mediators. In patients with bee venom allergy extensive molecular analyses might alert to potential unsuccessful outcome of SIT due to eventual allergen mismatch between bee venom and extract.25 Omalizumab should be considered as an additional therapy in high risk patients even if honey bee VIT is performed.

\section{Declarations}

Ethics approval and consent to participate: patient`s consent 
Consent for publication: patient`s consent

Availability of data and materials: not applicable

Competing interests: not applicable

Funding: not applicable

Authors' contributions: SM: manuscript writing, KR: manuscript writing, MB: manuscript revisions and writing (special focus CPR), PSG: : manuscript revisions and writing (special focus allergology), laboratory analyses

Acknowledgements: not applicable

Authors' information (optional): not applicable

\section{References}

1. Bilò MB, Bonifazi F. The natural history and epidemiology of insect venom allergy: clinical implications. Clin Exp Allergy 2009; 39: 1467-76.

2. Bilò MB. Anaphylaxis caused by Hymenoptera stings: from epidemiology to treatment. Allergy 2011; 66 Suppl 95: 35-7.

3. Schwartz LB, Metcalfe DD, Miller JS et al. Tryptase levels as an indicator of mast-cell activation in systemic anaphylaxis and mastocytosis. N Engl J Med 1987; 316: 1622-6.

4. Ruëff F, Wenderoth A, Przybilla B. Patients still reacting to a sting challenge while receiving conventional Hymenoptera venom immunotherapy are protected by increased venom doses. $J$ Allergy Clin Immunol. 2001 Dec;108(6):1027-32.

5. Pesek RD1, Lockey RF. Treatment of Hymenoptera venom allergy: an update. Curr Opin Allergy Clin Immunol. 2014;14(4):340-6

6. van Doormaal JJ, van der Veer E, van Voorst Vader PC et al. Tryptase and histamine metabolites as diagnostic indicators of indolent systemic mastocytosis without skin lesions. Allergy 2012; 67: 68390.

7. Pardanani A. Systemic mastocytosis in adults: 2013 update on diagnosis, risk stratification, and management. Am J Hematol 2013; 88: 612-24.

8. Valent P, Akin C, Arock $M$ et al. Definitions, criteria and global classification of mast cell disorders with special reference to mast cell activation syndromes: a consensus proposal. Int Arch Allergy Immunol 2012; 157: 215-25.

9. Fischer J, Teufel M, Feidt A et al. Tolerated wasp sting challenge improves health- related quality of life in patients allergic to wasp venom. J Allergy Clin Immunol 2013; 132: 489-90.

10. Oude Elberink JN, de Monchy JG, Kors JW et al. Fatal anaphylaxis after a yellow jacket sting, despite venom immunotherapy, in two patients with mastocytosis. J Allergy Clin Immunol 1997; 99: 153-4. 
11. Travers AH, Rea TD, Bobrow BJ, Edelson DP, Berg RA, Sayre MR, et al. Part 4: CPR overview: 2010 American Heart Association Guidelines for Cardiopulmonary Resuscitation and Emergency Cardiovascular Care. Circulation 2010; 122(18 Suppl 3):S676-84

12. Deakin CD, Nolan JP, Soar J, Sunde K, Koster RW, Smith GB, et al. European Resuscitation Council Guidelines for Resuscitation 2010 Section 4. Adult advanced life support. Resuscitation 2010;81(10):1305-52

13. Simons FE, Frew AJ, Ansotegui IJ et al. Risk assessment in anaphylaxis: current and future approaches. J Allergy Clin Immunol 2007; 120: S2-24.

14. Schwartz LB1, Atkins PC, Bradford TR, Fleekop P, Shalit M, Zweiman B. Release of tryptase together with histamine during the immediate cutaneous response to allergen.J Allergy Clin Immunol 1987. $198 ; 80(6): 850-5$

15. Triggiani M, Patella V, Staiano RI et al. Allergy and the cardiovascular system. Clin Exp Immunol 2008; 153 Suppl 1: 7-11.

16. Vadas P, Gold M, Perelman B et al. Platelet-activating factor, PAF acetylhydrolase, and severe anaphylaxis. N Engl J Med 2008; 358: 28-35.

17. Pesek RD, Lockey RF. Management of insect sting hypersensitivity: an update. Allergy Asthma Immunol Res 2013; 5: 129-37.

18. Canonica GW, Ansotegui IJ, Pawankar R et al. A WAO - ARIA - GA²LEN consensus document on molecular-based allergy diagnostics. World Allergy Organ J 2013; 6: 17.

19. Blank S, Seismann H, Michel Y et al. Api m 10, a genuine A. mellifera venom allergen, is clinically relevant but underrepresented in therapeutic extracts. Allergy 2011; 66: 1322-9.

20. Zuidmeer-Jongejan L, Fernández-Rivas M, Winter MG et al. Oil body-associated hazelnut allergens including oleosins are underrepresented in diagnostic extracts but associated with severe symptoms. Clin Transl Allergy. 2014;4(1):4-14

21. Kattan JD, Sicherer SH, Sampson HA. Clinical reactivity to hazelnut may be better identified by component testing than traditional testing methods. J Allergy Clin Immunol Pract 2014 Sep2(5):633-4

22. Gülsen A, Ruëff F, Jappe U. Omalizumab ensures compatibility to bee venom immunotherapy (VIT) after VIT-induced anaphylaxis in a patient with systemic mastocytosis. Allergol Select. $2021 \mathrm{Mar}$ $11 ; 5: 128-132$.

23. Kontou-Fili K, Filis Cl. Prolonged high-dose omalizumab is required to control reactions to venom immunotherapy in mastocytosis. Allergy 2009; 64: 1384-5.

24. Sokol KC, Ghazi A, Kelly BC, Grant JA. Omalizumab as a desensitizing agent and treatment in mastocytosis: a review of the literature and case report. J Allergy Clin Immunol Pract. 2014; 2(3):266-70

25. Frick M, Müller S, Bantleon F et al. rApi m 3 and rApi m 10 improve detection of honey bee sensitization in Hymenoptera venom-allergic patients with double sensitization to honey bee and yellow jacket venom. Allergy 2015 Dec;70(12):1665-8. 